Cite this: J. Mater. Chem. A, 2014, 2 , 8258

Received 16th December 2013 Accepted 12th March 2014

DOI: $10.1039 / c 3 t a 15224 c$

www.rsc.org/MaterialsA

\section{The beneficial effect of protic ionic liquids on the lithium environment in electrolytes for battery applications}

\begin{abstract}
Thomas Vogl, Sebastian Menne, Ruben-Simon Kühnel and Andrea Balducci*
This work reports the improved rate performance of ionic-liquid based lithium-ion batteries by replacement of the conventional aprotic ionic liquid (AIL) N-butyl- $N$-methylpyrrolidinium bis(trifluoromethanesulfonyl) imide (PYR 14 TFSI) by protic ionic liquids (PILs). Two model pyrrolidinium-TFSI PILs are synthesized and their mixtures with lithium bis(trifluoromethanesulfonyl)imide (LiTFSI) are characterized in terms of conductivity, viscosity and self-diffusion coefficients. Raman measurements show pronounced differences in terms of $\mathrm{TFSI}^{-}$coordination to $\mathrm{Li}^{+}$between the $\mathrm{AIL}$ and the PILs. $\mathrm{Li}^{+}$is coordinated by significantly fewer $\mathrm{TFSI}^{-}$anions in the investigated PILs, which is discussed as the likely cause for the much improved rate performance of lithium vanadium phosphate-based electrodes in these electrolytes.
\end{abstract}

\section{Introduction}

Lithium-ion batteries (LIBs) are nowadays one of the most important energy storage devices. LIBs are already dominating the consumer portable electronics market and have been indicated as the most promising electrochemical devices for the realization of hybrids and electric vehicles, as well as for advanced delocalized energy storage units. However, in order to be increasingly used in these new applications, the performance and safety of LIBs need to be improved. ${ }^{1}$

The commercially available LIBs contain electrolytes based on organic carbonates (e.g. ethylene carbonate (EC) and diethyl carbonate (DEC)). These electrolytes feature good conductivities, but since they are easily flammable and volatile, their use strongly reduces the safety as well as the temperature range of use of LIBs. For these reasons, in the last few years tremendous efforts have been focused on the development of alternative electrolytes. $^{1}$

Ionic liquids (ILs) are presently considered as one of the most promising candidates for the replacement of organic carbonates. $^{2-4}$ The main advantages of ILs towards organic carbonates are the high thermal, chemical and electrochemical stability as well as the negligible vapor pressure and the reduced flammability. ${ }^{2}$ So far, several aprotic ionic liquids (AILs) have been investigated as electrolytes for LIBs. The results of these studies showed that AILs can be successfully introduced in LIBs, and their use has beneficial effects on the safety as well as on the temperature range of use of these devices. ${ }^{3}$ Nevertheless, AILs are rather expensive and the performance of AIL-based

Westfälische Wilhelms-Universität, Institut für Physikalische Chemie-MEET, Corrensstraße 28/30, 48149 Münster, Germany. E-mail: andrea.balducci@ uni-muenster.de
LIBs still needs to be improved in order to be fully competitive with that of conventional electrolytes. Such improvement is particularly necessary for applications where high current densities are needed. In these applications the reduced lithiumion transport of AIL-based electrolytes, compared to that of conventional electrolytes, might significantly affect the rate performance of LIBs. In order to overcome this limitation, the use of mixtures of ionic liquids and organic electrolytes appears as a promising strategy., ${ }^{\mathbf{3 , 5}}$ Nevertheless, since such mixtures contain organic solvents, their safety is still lower compared to that of electrolytes containing only ILs. ${ }^{5,6}$ For these reasons, the development of new IL-based electrolytes with improved lithium-ion mobility compared to the one of present AIL-based electrolytes nowadays appears to be important for the introduction of ILs in LIBs. Taking into account the high cost of AILs, it would also be very beneficial to develop cheaper ILs, as they could be easily introduced in commercial devices.

Protic ionic liquids (PILs) are a subset of ILs and they are typically synthesized by neutralization reactions of a Brønsted acid (proton donor) and a Brønsted base (proton acceptor). ${ }^{7}$ PILs display all favorable properties of ILs, but they have the advantage of being easier to synthesize and cheaper compared to AILs. ${ }^{7,8}$ Clearly, these properties make them interesting candidates for use as electrolyte components for electrochemical devices. So far PILs have been successfully introduced as electrolytes for fuel cells and supercapacitors. ${ }^{8-11}$ In the case of fuel cells, PILs appear as interesting candidates for nonhumidifying intermediate-temperature fuel cells operating at a relatively high temperature. ${ }^{\mathbf{1 1}}$ In the case of supercapacitors, a recent study showed that the use of PILs allows the realization of devices with stable performance at different temperatures, but with lower operating voltage compared to that of conventional systems. ${ }^{10}$ 
It is worth noticing that the use of PILs as electrolytes for LIBs was not considered in the past. The availability of an acidic proton and its strong reactivity towards lithium were seen as an obstacle for the introduction of PILs into these devices, and consequently all efforts were focused on AILs. Nevertheless, we recently showed that in dry PILs the labile proton of the cation is not "free" and this cation is not subject to reversible protonation-deprotonation. ${ }^{10}$ We also proved that battery materials, e.g. lithium iron phosphate (LFP), can be used in combination with dry PILs without being subject to structural changes. ${ }^{12}$ Moreover, we showed that lithium-ion batteries containing PILbased electrolytes can be realized and that they display promising performance in terms of capacity and cycling stability. ${ }^{\mathbf{1 3}}$ Taking into account these results, PILs can therefore be regarded as a new class of electrolytes for LIBs. However, since only few studies have been dedicated to these electrolytes, a deeper investigation of PIL-based electrolytes is needed to understand the advantages and the limits related to their use in LIBs.

In this manuscript we report about the use of two pyrrolidinium-based PILs, pyrrolidinium bis(trifluoromethanesulfonyl)imide $\left(\mathrm{PYR}_{\mathrm{HH}} \mathrm{TFSI}\right)$ and $N$-butylpyrrolidinium bis(trifluoromethanesulfonyl)imide ( $\left.\mathrm{PYR}_{\mathrm{H} 4} \mathrm{TFSI}\right)$, in combination with lithium bis(trifluoromethanesulfonyl)imide (LiTFSI) as electrolytes for lithium-ion batteries. Initially the thermal stability, viscosity and conductivity of the two PIL-based electrolytes were considered. Afterwards, with the goal to get new insights into this class of electrolytes, the lithium mobility of these PIL-based electrolytes was investigated for the first time. Finally, the use of PIL-based electrolytes in combination with lithium vanadium phosphate (LVP) was evaluated. For all investigations, a comparison with the behavior of a pyrrolidinium-based AIL, 0.5 M LiTFSI in $N$-butyl- $N$-methylpyrrolidinium bis(trifluoromethanesulfonyl)imide ( $\left.\mathrm{PYR}_{14} \mathrm{TFSI}\right)$, was made.

\section{Experimental section}

The pyrrolidinium-based PILs, PYR $\mathrm{HH}_{\mathrm{H}}$ TFSI and PYR $_{\mathrm{H} 4}$ TFSI, were synthesized following a procedure similar to that described elsewhere. ${ }^{14}$ Pyrrolidine (Aldrich, >99\%) and 1-butylpyrrolidine (Aldrich, >98\%) were distilled directly before use. $\mathrm{HCl}(37 \%)$ and LiTFSI ( $3 \mathrm{M})$ were used as received. At the end of the synthesis, the obtained PILs were dried under vacuum at $60{ }^{\circ} \mathrm{C}$. The water content of the PILs was measured using coulometric Karl-Fischer titration, and was found to be lower than $10 \mathrm{ppm}$.

Thermogravimetric analysis was carried out using a Q5000 TGA instrument (TA Instruments). Ionic liquid samples with a typical weight of 20-30 mg were used during the experiments. The samples were heated from room temperature to $600{ }^{\circ} \mathrm{C}$ with a heating rate of $5{ }^{\circ} \mathrm{C} \min ^{-1}$ using nitrogen as a purge gas $\left(10 \mathrm{~cm}^{3} \mathrm{~min}^{-1}\right)$.

The conductivity and the viscosity of the prepared ILs were determined as reported in ref. 12. Self-diffusion coefficients were determined at $30{ }^{\circ} \mathrm{C}$ by pulsed field gradient (PFG)-NMR with an Avance III spectrometer (Bruker) equipped with a Diff30 probehead (Bruker) with selective RF-inserts for ${ }^{1} \mathrm{H},{ }^{7} \mathrm{Li}$ and ${ }^{19} \mathrm{~F}$ using the pulsed gradient stimulated echo (diffSte) sequence. ${ }^{15}$
Gradient pulse length, minimum gradient strength and diffusion time were set to $1 \mathrm{~ms}, 10 \mathrm{Gs} \mathrm{cm}^{-1}$ and $100 \mathrm{~ms}$ for the ${ }^{1} \mathrm{H}$ and ${ }^{19} \mathrm{~F}$ measurements and $3 \mathrm{~ms}, 20 \mathrm{Gs} \mathrm{cm}^{-1}$ and $70 \mathrm{~ms}$ for the ${ }^{7} \mathrm{Li}$ measurements. The maximum gradient strength was chosen in order to obtain a strong signal damping. A typical maximum gradient strength was $800 \mathrm{Gs} \mathrm{cm}^{-1}$. The fitting was done with TopSpin 3.0 (Bruker) software using the Stejskal-Tanner equation. All proton signals were considered for the determination of the pyrrolidinium diffusion coefficients.

Raman spectra were recorded with a software-controlled Bruker Vertex 70 RAM II FT-Raman device with a HeNe-laser, a $\mathrm{CaF}_{2}$ beam splitter and a LN-Ge diode as a detector. The number of scans was set to 512 and the laser power was set to $500 \mathrm{~mW}$. The Raman spectra were recorded at room temperature.

Lithium vanadium phosphate (LVP) composite electrodes were prepared as in ref. 16. The composition of the electrodes was $70 \mathrm{wt} \%$ of the active material LVP, $20 \mathrm{wt} \%$ of the conductive agent Super $\mathrm{C} 65 \AA$ and $10 \mathrm{wt} \%$ of polyvinylidene fluoride (PVDF) as a binder. The electrode mass loading was $1 \mathrm{mg} \mathrm{cm}^{-2}$ and the electrode area was $1.13 \mathrm{~cm}^{2}$. All the electrochemical tests were carried out with 3 -electrode Swagelok ${ }^{\circledR}$ type cells. The cells were assembled in an argon-filled glove box with oxygen and water contents lower than 1 ppm. LVP-based electrodes were used as working electrodes, a silver wire was used as the pseudo-reference electrode and an oversized activated carbon-based electrode was used as the counter electrode. For all experiments, a 3-layered non-woven separator (Freudenberg) drenched with $100 \mu \mathrm{L}$ of electrolyte was used.

Electrochemical impedance spectroscopy (EIS) was performed at $30{ }^{\circ} \mathrm{C}$ using a VMP multichannel potentiostatic-galvanostatic system (Biologic Science Instruments, France). Prior to the measurements, 20 galvanostatic cycles with a current corresponding to a rate of $1 \mathrm{C}$ were carried out. Afterward, impedance spectra were recorded at the fully lithiated state (fully discharged) and in a partially lithiated state (at the first plateau of the discharge) in the frequency range between $1 \mathrm{MHz}$ and $10 \mathrm{mHz}$ with an alternating voltage of $5 \mathrm{mV}$.

The electrochemical measurements were carried out using a MACCOR Series 4000 battery tester. Constant current cycling (CC) was carried out at $30{ }^{\circ} \mathrm{C}$ using current densities ranging from $1 \mathrm{C}$ to $20 \mathrm{C}$ taking into account the theoretical capacity of LVP of $131 \mathrm{~mA} \mathrm{~h} \mathrm{~g}^{-1}$ when cycled between 3.0 and $4.3 \mathrm{~V}$ vs. $\mathrm{Li} / \mathrm{Li}^{+}$.

\section{Results and discussion}

The chemical-physical properties of ILs are dramatically affected by the presence of impurities and the presence of water. $^{2-4,13}$ As mentioned in the Introduction, a pre-requisite for the utilization of PILs in LIBs is the use of dry electrolytes (with water content lower than $20 \mathrm{ppm}$ ) as under these conditions the labile proton(s) of the cation is not "free" and it does not interfere with the lithiation-delithiation process of the LIB electrodes. ${ }^{\mathbf{1 0 , 1 3}}$ As indicated in the Experimental section, the investigated PILs displayed water contents below $10 \mathrm{ppm}$, and therefore they both appear suitable for use as electrolytes for LIBs. 


\subsection{Thermal stability}

Fig. 1 compares the thermal stability of the PIL-based electro-

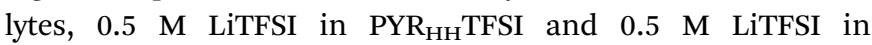
$\mathrm{PYR}_{\mathrm{H} 4}$ TFSI, with that of the AIL-based electrolyte 0.5 M LiTFSI in PYR $_{14}$ TFSI. LiTFSI was selected as lithium salt since it has the same anion of all ILs. As shown, both PIL-based electrolytes are thermally stable up to $250{ }^{\circ} \mathrm{C}$. Between the two, the electrolyte containing PYR $_{\mathrm{HH}}$ TFSI appears slightly more stable than the one containing $\mathrm{PYR}_{\mathrm{H} 4}$ TFSI. The electrolyte containing the AIL PYR $_{14}$ TFSI, on the other hand, appears more stable as a significant weight loss begins at more than $300^{\circ} \mathrm{C}$. These results clearly indicate that PIL-based electrolytes display thermal stability significantly higher than that of conventional organic electrolytes. ${ }^{6}$

\subsection{Conductivity and viscosity}

Fig. 2 compares the conductivity and viscosity of two PIL-based electrolytes, 0.5 M LiTFSI in PYR $\mathrm{HH}_{\mathrm{H}}$ TFSI and 0.5 M LiTFSI in PYR $_{\mathrm{H} 4}$ TFSI, with those of the AIL-based electrolyte $0.5 \mathrm{M}$ LiTFSI in PYR 14 $_{14}$ TFSI. As shown in Fig. 2a, the two PIL-based electrolytes display similar conductivity in the investigated temperature range. At $30{ }^{\circ} \mathrm{C}$ they both display conductivity in the order of $2 \mathrm{mS} \mathrm{cm}^{-1}$, which increases to $7 \mathrm{mS} \mathrm{cm}^{-1}$ at $80^{\circ} \mathrm{C}$. These values are comparable with the ones shown by the electrolyte 0.5 M LiTFSI in PYR P $_{14}$ TFSI in the same temperature range. It is worth noticing that it is often reported in the literature that PILs display higher conductivity compared to AILs. ${ }^{17}$ Nevertheless, most of the reported values refer to PILs containing high amounts of water and, most likely, the presence of water is the origin of this high conductivity. ${ }^{13}$ As shown above, when dry PILs are considered a marked difference in terms of ionic conductivity between PILs and AILs cannot be observed. Fig. 2 b shows the variation of the viscosity over the temperature for two PIL-based electrolytes. As for the conductivity, also the viscosity of the PIL-based electrolytes was comparable to that of $0.5 \mathrm{M}$ LiTFSI in PYR $_{14}$ TFSI. Nevertheless, it is interesting to note that at $30{ }^{\circ} \mathrm{C}$ the electrolyte 0.5 M LiTFSI in PYR HH $_{\mathrm{H}}$ TFSI displays a viscosity of $130.1 \mathrm{mPa}$,

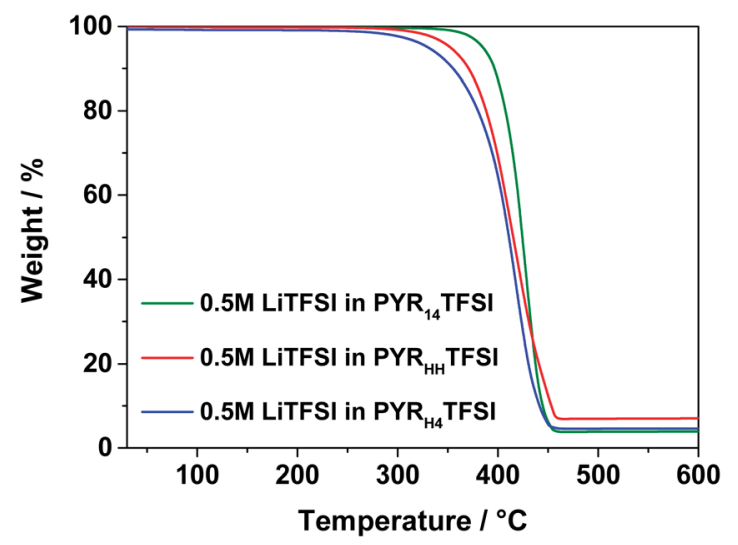

Fig. 1 Thermal stability of the electrolytes $0.5 \mathrm{M} \mathrm{LiTFSI}$ in PYR $\mathrm{HH}_{\mathrm{H}}$ TFSI, $0.5 \mathrm{M}$ LiTFSI in PYR $\mathrm{H}_{4}$ TFSI and 0.5 M LiTFSI in PYR ${ }_{14}$ TFSI.
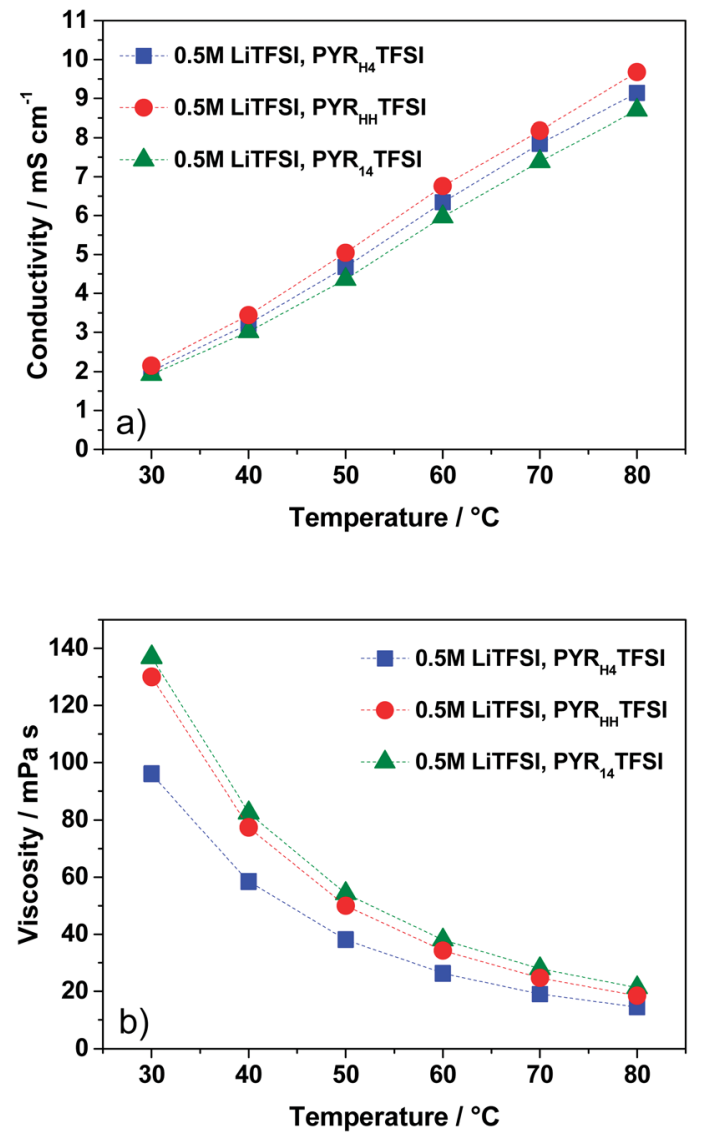

Fig. 2 Conductivity (a) and viscosity (b) of the electrolytes 0.5 M LiTFSI in PYR $\mathrm{HH}_{\mathrm{H}}$ TFSI, 0.5 M LiTFSI in PYR $\mathrm{H}_{4}$ TFSI and 0.5 M LiTFSI in PYR ${ }_{14}$ TFSI.

which is higher than the one of $0.5 \mathrm{M}$ LiTFSI in PYR $_{\mathrm{H} 4}$ TFSI (96.0 $\mathrm{mPa}$ ). Taking into account the conductivities of the two electrolytes, the higher viscosity of $\mathrm{PYR}_{\mathrm{HH}}$ TFSI compared to $\mathrm{PYR}_{\mathrm{H} 4}$ TFSI appears somehow surprising. This behavior might be originated from the different size of the cations of these PILs as well as from their different interactions with the TFSI $^{-}$ anion. The $\mathrm{PYR}_{\mathrm{HH}}{ }^{+}$cation is obviously smaller than the $\mathrm{PYR}_{\mathrm{H} 4}{ }^{+}$ cation and this characteristic might be responsible for the higher conductivity of the electrolyte containing PYR $_{\mathrm{HH}} \mathrm{TFSI}$ compared to the one containing PYR $_{\mathrm{H} 4}$ TFSI. At the same time, as it is less shielded, the cation $\mathrm{PYR}_{\mathrm{HH}}{ }^{+}$is probably subject to a stronger interaction with the anion $\mathrm{TFSI}^{-}$compared to the cation $\mathrm{PYR}_{\mathrm{H} 4}{ }^{+}$, and this interaction might explain the higher viscosity of the electrolyte containing PYR $_{\mathrm{HH}}$ TFSI compared to the one containing PYR $_{\mathrm{H} 4}$ TFSI. At higher temperatures, the viscosity of both electrolytes decreases and becomes more similar. At $80^{\circ} \mathrm{C}$, both electrolytes have viscosities in the range of 14.4-18.0 mPa s.

The temperature dependence of the measured conductivities and viscosities did not show Arrhenius-like behavior, but it could be well described by the Vogel-Tammann-Fulcher (VTF) model. Fig. 3 shows the VTF plots for conductivity and viscosity of all electrolytes. The VTF-equations (1) and (2) are shown below and the VTF fitting parameters are reported in Table 1. 

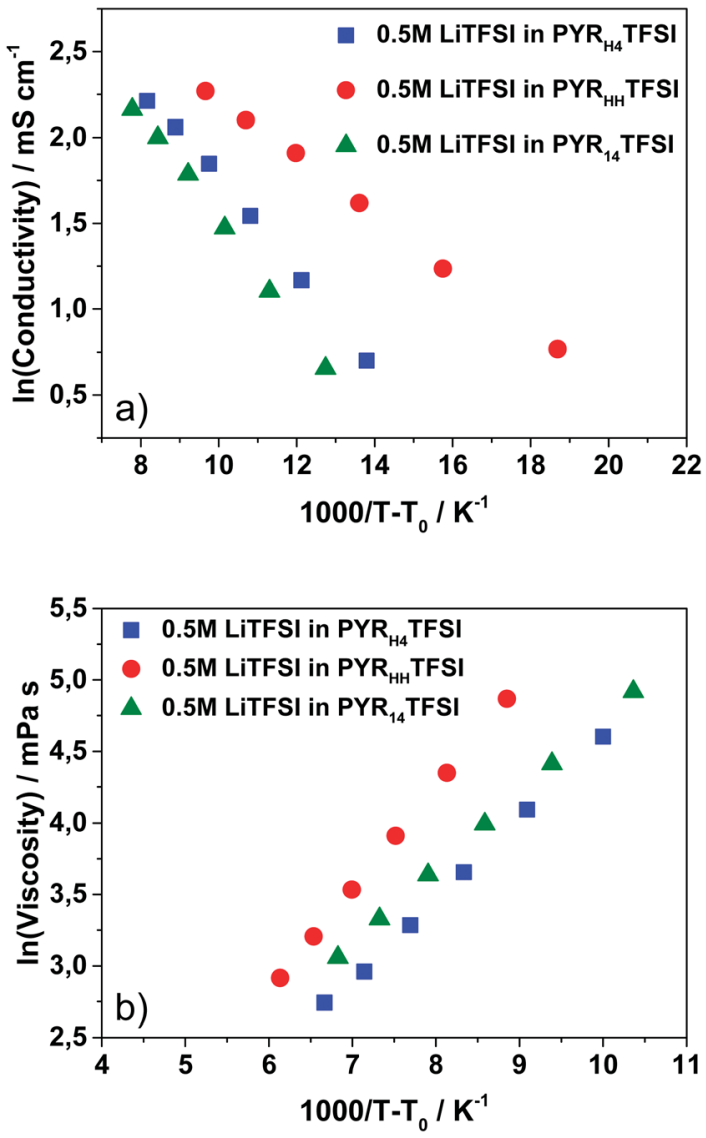

Fig. 3 VTF plots of conductivity (a) and viscosity (b) of the electrolytes 0.5 M LiTFSI in PYR $\mathrm{HH}_{\mathrm{H}}$ TFSI, 0.5 M LiTFSI in PYR $\mathrm{H}_{4}$ TFSI and 0.5 M LiTFSI in $\mathrm{PYR}_{14} \mathrm{TFSI}$.

$$
\begin{aligned}
& \sigma=\sigma_{0} \exp \left[\frac{-B_{\mathrm{c}}}{T-T_{0 \mathrm{c}}}\right] \\
& \eta=\eta_{0} \exp \left[\frac{-B_{\mathrm{v}}}{T-T_{0 \mathrm{v}}}\right]
\end{aligned}
$$

\subsection{Diffusion measurements}

As shown above, the conductivity and viscosity of dry pyrrolidinium-based PILs are rather similar to those of pyrrolidiniumbased AILs. As mentioned in the Introduction, one of the main limitations of AIL-based electrolytes is their reduced lithiumion mobility compared to the one of conventional electrolytes. The ion mobility as well as the lithium coordination of AILs has already been investigated in the past. ${ }^{\mathbf{1 8 , 1 9}}$ Nevertheless, to the

Table 1 VTF fitting parameters for the conductivity and viscosity of

\begin{tabular}{|c|c|c|c|c|c|c|}
\hline & \multicolumn{3}{|c|}{ Conductivity } & \multicolumn{3}{|c|}{ Viscosity } \\
\hline & $T_{0 \mathrm{c}} / \mathrm{K}$ & $\sigma_{0} / \mathrm{mS} \mathrm{cm}^{-1}$ & $B_{\mathrm{c}} / \mathrm{K}$ & $T_{\mathrm{Ov}} / \mathrm{K}$ & $\eta_{0} / \mathrm{mPa} \mathrm{s}$ & $B_{\mathrm{v}} / \mathrm{K}$ \\
\hline PYR $_{\mathrm{H} 4}$ TFSI & 230.5 & 49.49 & 168.0 & 203.0 & 0.356 & -562.7 \\
\hline PYR $_{H H}$ TFSI & 249.5 & 84.64 & 268.9 & 190.0 & 0.225 & -718.5 \\
\hline PYR $_{14}$ TFSI & 224.5 & 95.94 & 305.0 & 206.5 & 0.587 & -526.9 \\
\hline
\end{tabular}
solutions of $0.5 \mathrm{M} \mathrm{LiTFSI}$ in ionic liquids best of our knowledge, so far the $\mathrm{Li}^{+}$mobility as well as the lithium coordination in PIL-based electrolytes has not been investigated. Considering the importance of these properties for the development of advanced IL-based electrolytes for LIBs, we therefore investigated both of them. Table 2 lists self-diffusion coefficients and the dissociation degree for the investigated electrolytes. Only relatively small differences in selfdiffusion coefficients between the different electrolytes were found. This was surprising; it was expected that reducing the size of the pyrrolidinium cation by substituting one or both of the alkyl chains of $\mathrm{PYR}_{14}{ }^{+}$with a hydrogen atom would accelerate its diffusion, in line with the increasing conductivity (Fig. 2a). Substituting the methyl group of $\mathrm{PYR}_{14}{ }^{+}$by a hydrogen atom had the opposite effect: all self-diffusion coefficients became slightly smaller, although the conductivity of this electrolyte was found to be higher than the one of PYR ${ }_{14}$ TFSI0.5 M LiTFSI. This can be understood by considering that it cannot be distinguished between un-paired and aggregated ions with the NMR measurements. Hence, also ion pairs and other neutral aggregates can contribute to the measured selfdiffusion coefficients. These neutral species do not contribute to the electrolyte conductivity, which is consequently lowered by their presence. The Nernst-Einstein equation (eqn (3)) allows the calculation of an apparent conductivity treating all diffusing species as charge carriers contributing to conductivity:

$$
\sigma_{\mathrm{NMR}}=\frac{e^{2}}{k T} \sum N_{\mathrm{V}, \mathrm{i}} D_{\mathrm{i}}
$$

here $e$ is the elementary charge, $k$ is the Boltzmann constant, $T$ is the absolute temperature, $N_{\mathrm{V}, \mathrm{i}}$ is the number density of ion $\mathrm{i}$ (derived from the concentrations of the ions and the density of the electrolyte) and $D_{\mathrm{i}}$ is the self-diffusion coefficient of ion i. The ratio between the measured conductivity $\sigma_{\mathrm{AC}}$ and $\sigma_{\mathrm{NMR}}$ can be interpreted as the dissociation degree $\Delta$, i.e., the higher is the dissociation degree the more ions of the electrolyte contribute to its conductivity, although it should not be understood in a strict sense due to the complexity of the ion interactions in ILs. The dissociation degree of $87 \%$ calculated for PYR $_{\mathrm{H} 4}$ TFSI- $0.5 \mathrm{M}$ LiTFSI indicates that this electrolyte is more dissociated than the electrolyte PYR $_{14}$ TFSI-0.5 M LiTFSI with a dissociation degree of $74 \%$. This higher degree of dissociation explains the higher conductivity of the $\mathrm{PYR}_{\mathrm{H} 4}$ TFSI-0.5 M LiTFSI electrolyte compared to PYR $_{14}$ TFSI-0.5 M LiTFSI. Substituting also the butyl chain of $\mathrm{PYR}_{14}{ }^{+}$by a hydrogen atom slightly increased all the self-diffusion coefficients. The $\mathrm{PYR}_{\mathrm{HH}}{ }^{+}$cation is significantly smaller than the $\mathrm{PYR}_{14}{ }^{+}$cation. Hence, a pronounced

Table 2 Self-diffusion coefficients $\left(D_{\mathrm{i}}\right)$ and dissociation degree $(\Delta)$ of $0.5 \mathrm{M}$ LiTFSI solutions of PYR 14 TFSI, PYR ${ }_{4}$ TFSI and $\mathrm{PYR}_{\mathrm{HH}}$ TFSI, respectively, at $30^{\circ} \mathrm{C}$

\begin{tabular}{lllll}
\hline & \multicolumn{2}{c}{$D_{\mathrm{i}} / 10^{-12} \mathrm{~m}^{2} \mathrm{~s}^{-1}$} & & \\
\cline { 2 - 4 } Ionic liquid & $\mathrm{PYR}_{X Y}{ }^{+}$ & $\mathrm{TFSI}^{-}$ & $\mathrm{Li}^{+}$ & $\Delta=\frac{\sigma_{\mathrm{AC}}}{\sigma_{\mathrm{NMR}}}$ \\
\hline $\mathrm{PYR}_{14}$ TFSI & 12.0 & 8.1 & 5.3 & $74 \%$ \\
PYR $_{\mathrm{H} 4}$ TFSI & 10.5 & 6.9 & 4.7 & $87 \%$ \\
PYR $_{\mathrm{HH}}$ TFSI & 12.2 & 9.1 & 5.7 & $59 \%$
\end{tabular}


increase of its diffusivity could be expected. However, the corresponding self-diffusion coefficient increased only from $12.0 \times$ $10^{-12}$ to $12.2 \times 10^{-12} \mathrm{~m}^{2} \mathrm{~s}^{-1}$. At the same time, the dissociation degree decreased from $74 \%$ to $59 \%$. Taking these two findings into account, it can be concluded that $\mathrm{TFSI}^{-}$is interacting much stronger with $\mathrm{PYR}_{\mathrm{HH}}{ }^{+}$than with $\mathrm{PYR}_{14}{ }^{+}$. The positive charge at the nitrogen atom is much less shielded in the case of $\mathrm{PYR}_{\mathrm{HH}}{ }^{+}$ than in the case of $\mathrm{PYR}_{14}{ }^{+}$. As a result, ion pairing between $\mathrm{PYR}_{\mathrm{HH}}{ }^{+}$and TFSI $^{-}$can be expected, as expressed by the lower dissociation degree. The same is most likely true for $\mathrm{PYR}_{\mathrm{H} 4}{ }^{+}$, but to a lower extent. As a consequence of the differences in $\mathrm{PYR}_{X Y}{ }^{+}$ TFSI $^{-}$interaction, the viscosity of the electrolyte containing PYR $_{\mathrm{HH}}$ TFSI is higher than that of the electrolyte containing $\mathrm{PYR}_{\mathrm{H} 4}$ TFSI (as reported above). The lower self-diffusion coefficient for the smaller $\mathrm{PYR}_{\mathrm{H} 4}{ }^{+}$cation compared to the bigger $\mathrm{PYR}_{14}{ }^{+}$cation, although at the same time the viscosity was lower for the $\mathrm{PYR}_{\mathrm{H} 4}$ TFSI-based electrolyte, indicates that also in the case of $\mathrm{PYR}_{\mathrm{H} 4}{ }^{+}$the interaction with $\mathrm{TFSI}^{-}$is more pronounced thus increasing the effective size of the diffusing $\mathrm{PYR}_{\mathrm{H} 4}{ }^{+}$ species. It is important to mention that the increased interaction between $\mathrm{PYR}_{X Y}{ }^{+}$and $\mathrm{TFSI}^{-}$should also change the interaction between $\mathrm{Li}^{+}$and $\mathrm{TFSI}^{-}$.

\subsection{Lithium-ion environment}

To investigate the different ion-ion interactions in AIL- and PILbased electrolytes in more detail, Raman spectra were recorded for all electrolytes. A characteristic (and strong) signal relative to the interaction of TFSI $^{-}$with other ions is the mode, found at a wavenumber of $742 \mathrm{~cm}^{-1}$, which is generated by the expansion/ contraction of the whole TFSI $^{-}$anion. In the literature, this signal is typically assigned to non-coordinating or "free" TFSI $^{-}$anions. ${ }^{20-22}$ Coordinating (or interacting) TFSI $^{-}$anions generate an additional signal, which is shifted to higher wavenumbers in the Raman spectra (e.g. lithium ion coordinating $\mathrm{TFSI}^{-}, c a$. $\left.748 \mathrm{~cm}^{-1}\right) .{ }^{23,24}$ Table 3 lists the assigned peak positions for the two modes (non-coordinating/coordinating) observed in the investigated electrolytes. The non-coordinating TFSI $^{-}$(TFSI ${ }_{\text {non }}$ ) mode for 0.5 M LiTFSI in PYR $_{14}$ TFSI was found to be at $742.4 \mathrm{~cm}^{-1}$. This value is in agreement with values reported in the literature for "free" TFSI-anions. ${ }^{23}$ In the case of the PIL-based electrolytes, the TFSI $_{\text {non }}$ mode was shifted to higher wavenumbers, indicating the presence of relatively important ion-ion interactions between the IL-cations and $\mathrm{TFSI}^{-}$in these electrolytes. The presence of these

Table 3 Raman peak positions of non-coordinating $\mathrm{TFSI}^{-}$(TFSI $_{\text {non }}$ ) and $\mathrm{Li}^{+}$coordinating $\mathrm{TFSI}^{-}\left(\mathrm{TFSI}_{\text {coor }}\right.$ ), fraction of the corresponding peak area of $\mathrm{TFSI}_{\text {coor }}$ compared to the sum of the peak areas of $\mathrm{TFSI}_{\text {coor }}$ and $\mathrm{TFSI}_{\text {non }}\left(\mathrm{TFSI}_{\text {coor }}\right)$ and calculated $\mathrm{Li}^{+}$coordination number $n$ for $0.5 \mathrm{M}$ LiTFSI solutions of PYR ${ }_{14}$ TFSI, PYR $\mathrm{H}_{4}$ TFSI and PYR $\mathrm{H}_{\mathrm{H}}$ TFSI, respectively

\begin{tabular}{lllll}
\hline Ionic liquid & TFSI $_{\text {non }} / \mathrm{cm}^{-1}$ & TFSI $_{\text {coor }} / \mathrm{cm}^{-1}$ & TFSI $_{\text {coor }} / \%$ & $\begin{array}{l}n \text { in }\left(\mathrm{Li}^{+}\right) \\
\left(\mathrm{TFSI}^{-}\right)_{n}\end{array}$ \\
\hline PYR $_{14}$ TFSI & 742.4 & 748.5 & 27.04 & 1.9 \\
PYR $_{\text {H4 }}$ TFSI & 744.6 & 749.0 & 16.47 & 1.2 \\
PYR $_{\text {HH }}$ TFSI & 745.6 & 749.7 & 9.12 & 0.4
\end{tabular}

interactions is also confirmed by the shift of the peak wavenumbers corresponding to the $\mathrm{Li}^{+}$coordinating $\mathrm{TFSI}^{-}$(TFSI ${ }_{\text {coor }}$ ) to higher wavenumbers, which is more marked in the case of the two PIL-based electrolytes than for the electrolytes containing PYR $_{14}$ TFSI (Fig. 4). In order to evaluate the ratio between coordinated and free ionic species, a useful method is to compare the areas of these species in the Raman spectra. ${ }^{24}$

Fig. 4 shows the Raman spectra of the three electrolytes and the fitted areas for non-coordinating and coordinating TFSI $^{-}$. From the figures it can be seen that the areas of TFSI coor $_{\text {became }}$ smaller moving from PYR $_{14}$ TFSI to PYR ${ }_{\mathrm{H} 4}$ TFSI to PYR $\mathrm{HH}_{\mathrm{TFSI}}$. Accordingly, the fraction of $\mathrm{TFSI}_{\text {coor }}$ decreases (as indicated in Table 3), following the same trend. When the lithium coordination number in the investigated electrolyte is calculated, ${ }^{19}$ the different behavior of PILs and AILs became evident. As indicated in Table 3, in PYR 14 TFSI the $\mathrm{Li}^{+}$ions are well coordinated by $c a .2$ TFSI $^{-}$anions, while in $\mathrm{PYR}_{\mathrm{HH}}$ TFSI the $\mathrm{Li}^{+}$ions are coordinated by only $0.4 \mathrm{TFSI}^{-}$anions. Taking this result into account, it is reasonable to suppose that the less shielded PIL-cations restrain TFSI $^{-}$anions from coordinating to $\mathrm{Li}^{+}$ions. As a consequence of this "cation competition", the $\mathrm{Li}^{+}$ion in PIL-based electrolytes becomes significantly less coordinated compared to AILs. ${ }^{25}$

The results reported above clearly indicate that AIL- and PILbased electrolytes, although they display similar ionic conductivity and viscosity, display marked differences in terms of ion coordination. As shown by the Raman study, the interaction between $\mathrm{Li}^{+}$ and TFSI $^{-}$is much stronger in the AIL-based electrolyte than in the case of the PIL-based ones. The lower interaction in the latter electrolytes is due to the presence of less shielded cations, which "compete" with the $\mathrm{Li}^{+}$ion for the coordination of the anion. Nevertheless, it is very interesting to notice that the differences in coordination did not lead to pronounced changes in the lithium diffusion coefficient, as indicated by the NMR measurement. Taking these findings into account, the main difference between AIL- and PIL-based electrolytes appears to be the lithium environment (see Fig. 4). $\mathrm{Li}^{+}$is certainly more loosely coordinated in the investigated PILs than in the investigated AILs. This difference appears extremely interesting as it could have a positive impact on the power performance of lithium-ion batteries.

\subsection{Electrochemical characterization}

With the aim to verify the latter point, we investigated the performance of LVP-based electrodes in the three investigated
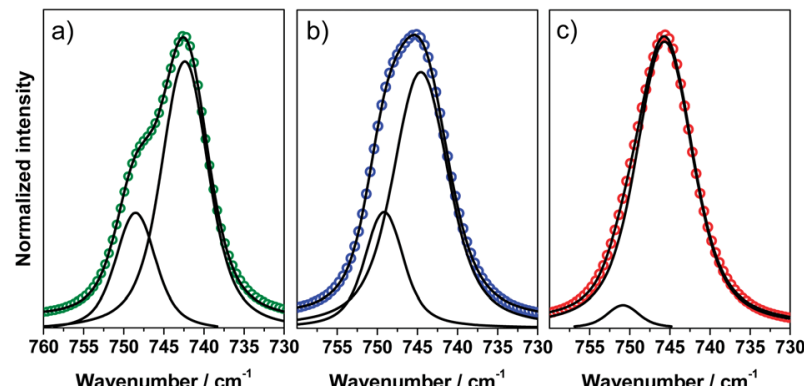

Wavenumber $/ \mathrm{cm}^{-1}$

Wavenumber $/ \mathrm{cm}^{-1}$

Fig. 4 Raman spectra in the spectral range from $730-760 \mathrm{~cm}^{-1}$ of 0.5 M LiTFSI in PYR 14 TFSI (a), PYR $\mathrm{H}_{4}$ TFSI (b) and PYR $\mathrm{HH}^{T}$ TFSI (c). 
electrolytes. Specifically, we carried out a C-rate test, at $30{ }^{\circ} \mathrm{C}$, using currents ranging from $1 \mathrm{C}$ to $20 \mathrm{C}$. In order to have a more exhaustive comparison, also the conventional electrolyte $1 \mathrm{M}$ $\mathrm{LiPF}_{6}$ in EC:DMC was used for this test. As shown in Fig. 5, when a current density corresponding to $1 \mathrm{C}$ was applied, LVP cathodes displayed in both PIL-based electrolytes specific capacities in the order of $120 \mathrm{~mA} \mathrm{~h} \mathrm{~g}{ }^{-1}$. These values are close to the theoretical capacity of LVP $\left(131 \mathrm{~mA} \mathrm{~h} \mathrm{~g}^{-1}\right.$ between $3.0 \mathrm{~V}$ and 4.3 $\mathrm{V} v$ s. $\mathrm{Li} / \mathrm{Li}^{+}$) and they were comparable to those observed for the same electrodes in the conventional electrolyte. This capacity was $10 \%$ higher than that observed for $0.5 \mathrm{M}$ LiTFSI in PYR $_{14}$ TFSI (110 $\left.\mathrm{mA} \mathrm{h} \mathrm{g}^{-1}\right)$. When the C-rate was increased, the differences between AIL- and PIL-based electrolytes became even more severe. At $5 \mathrm{C}$, the LVP electrodes used in combination with PIL-based electrolytes displayed 30\% higher capacity than those cycled with the AIL-based electrolyte. At $20 \mathrm{C}$, the LVP electrode cycled with 0.5 M LiTFSI in PYR $_{14}$ TFSI delivered a capacity of $c a .20 \mathrm{~mA} \mathrm{~h} \mathrm{~g}{ }^{-1}$, while the ones cycled with $0.5 \mathrm{M}$ LiTFSI in PYR ${ }_{\mathrm{HH}}$ TFSI and 0.5 M LiTFSI in PYR $_{\mathrm{H} 4}$ TFSI displayed a capacity three times higher, in the order of $55-60 \mathrm{~mA} \mathrm{~h}^{-1}$. As shown in the figure, at high C-rates (higher than $5 \mathrm{C}$ ) the performance of all IL-based electrolytes was still lower than the one obtained with the conventional electrolyte. Nevertheless, the results of this investigation indicate that the presence of loosely coordinated $\mathrm{Li}^{+}$ions in PIL-based electrolytes, compared to that in AIL-based electrolytes, has dramatic consequences on the electrode performance.

In order to understand the reasons of the different behavior of the LVP electrodes in the investigated electrolytes, impedance spectra were recorded at the fully lithiated state (after full discharge) and in a partially lithiated state (at the first plateau of the discharge). The latter state was selected in order to have indication about the charge-transfer resistance in the electrolytes. Since the two PILs display similar behavior, only the electrolyte 0.5 M LiTFSI in PYR $_{\mathrm{H} 4}$ TFSI was used for this test. As shown in Fig. 6, in all electrolytes the impedance spectra of the LVP electrodes in the fully lithiated state presented a semicircle

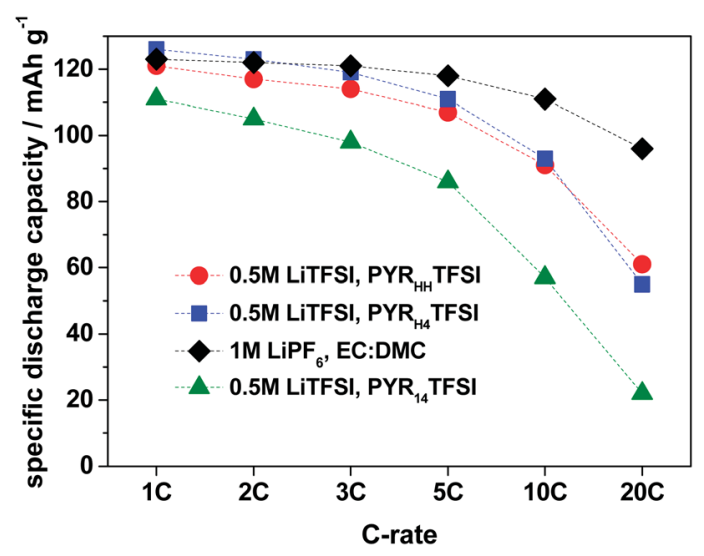

Fig. 5 Comparison of the specific discharge capacity of LVP electrodes during charge-discharge tests carried out at $\mathrm{C}$-rates ranging from $1 \mathrm{C}$ to $20 \mathrm{C}$ in the electrolytes $0.5 \mathrm{M} \mathrm{LiTFSI}$ in PYR $\mathrm{HH}_{\mathrm{HH}}$ TFSI, $0.5 \mathrm{M}$ LiTFSI in $\mathrm{PYR}_{\mathrm{H} 4}$ TFSI, $0.5 \mathrm{M} \mathrm{LiTFSI}$ in $\mathrm{PYR}_{14}$ TFSI and $1 \mathrm{M} \mathrm{LiPF}_{6}$ in EC:DMC at $30^{\circ} \mathrm{C}$ at high-medium frequency, followed by a diffusion part at low frequency. ${ }^{26}$ As expected, the charge-transfer resistance observed in the conventional electrolyte was the lowest (Fig. 6b) which was also shown in the literature before. ${ }^{27}$ Both IL-based electrolytes show higher charge-transfer resistance due to their lower conductivity. Nevertheless, it is important to notice that the overall resistance observed in the PIL-based electrolytes (Fig. 6d) was lower than that observed in the AIL-based one (Fig. 6f). The impedance spectra of the LVP electrodes in the partially lithiated state were significantly different. In all electrolytes a second semicircle, located in the medium frequency region, appeared. The presence of this additional semicircle in the partially lithiated state is correlated with the charge-transfer resistance associated with the lithium insertion into the LVP structure. ${ }^{26,28}$ Also in this case, the charge-transfer resistance observed in the conventional electrolyte was the lowest. However, it is very interesting to notice the remarkable difference between the PIL-based electrolyte and the AIL-based one. As shown, the semicircle in the case of $0.5 \mathrm{M}$ LiTFSI in $\mathrm{PYR}_{14}$ TFSI was significantly larger than the one of $0.5 \mathrm{M}$ LiTFSI in PYR $_{\mathrm{H} 4}$ TFSI, indicating the presence of a much higher charge transfer resistance in the AIL. Taking into account the Raman results reported above, it appears reasonable to suppose that the lithium desolvation process is easier in PILs than in AILs, and that this difference could be the origin of the different charge-transfer resistance between these two types of ILs. As shown in Fig. 5, this different charge-transfer resistance affects
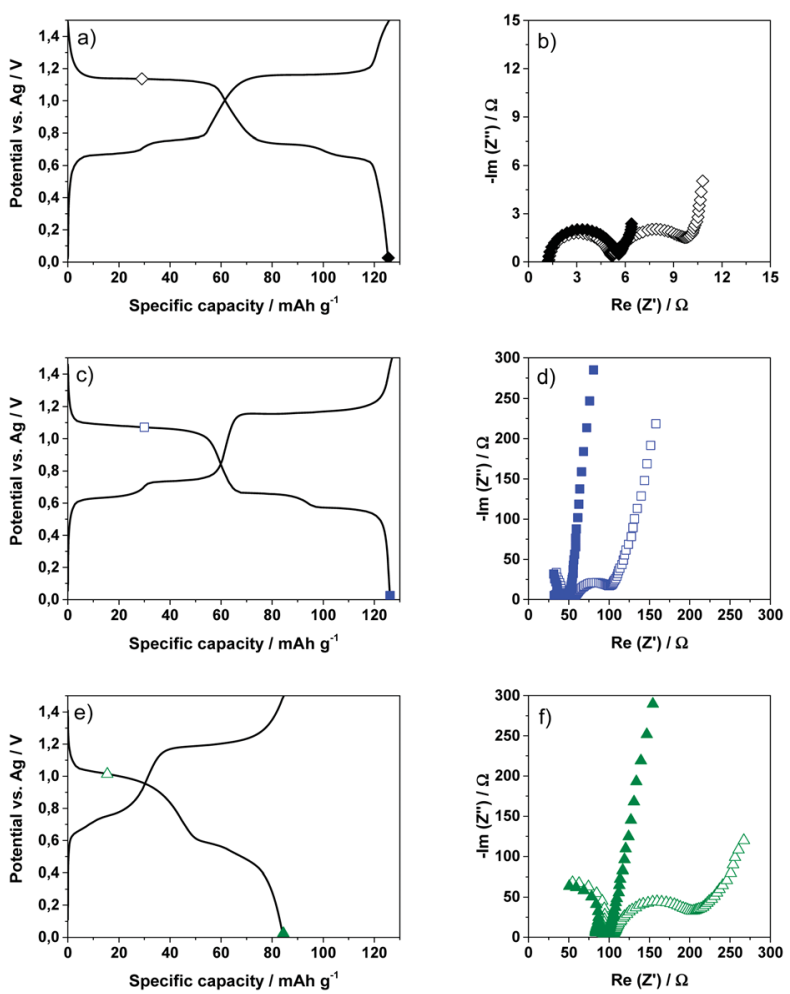

Fig. 6 Comparison of the voltage profile and impedance spectra at the fully lithiated and partially delithiated states of the LVP electrode in the electrolytes $1 \mathrm{M} \mathrm{LiPF}_{6}$ in EC:DMC at $30{ }^{\circ} \mathrm{C}$ (a and b), $0.5 \mathrm{M} \mathrm{LiTFSI} \mathrm{in}$ PYR $_{\mathrm{H}_{4}}$ TFSI ( $c$ and d) and $0.5 \mathrm{M}$ LiTFSI in PYR ${ }_{14}$ TFSI (e and f). 


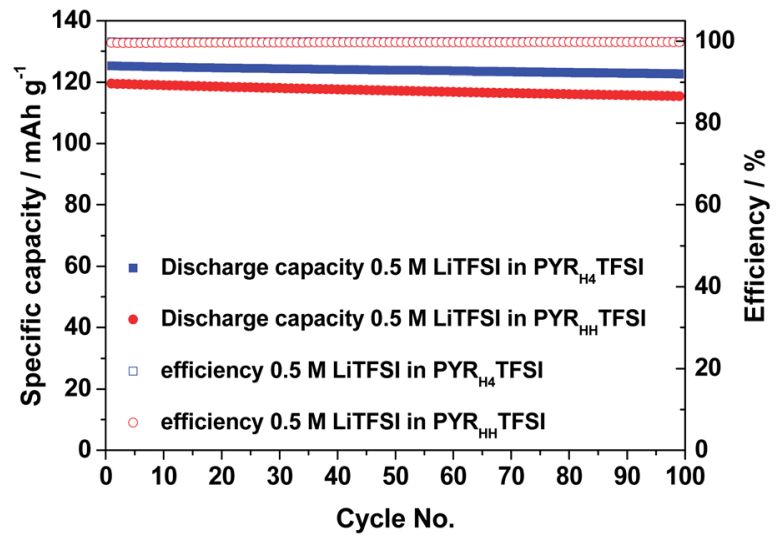

Fig. 7 Cycling stability of LVP electrodes in the electrolytes $0.5 \mathrm{M}$ LiTFSI in PYR $\mathrm{HH}_{\mathrm{H}}$ TFSI and $0.5 \mathrm{M}$ LiTFSI in PYR ${ }_{\mathrm{H} 4}$ TFSI at $30{ }^{\circ} \mathrm{C}$. The constant current cycling was carried out at a $\mathrm{C}$-rate of $1 \mathrm{C}$.

significantly the behavior of the electrodes during tests at high current densities and, most likely, is one of the main responsible reasons for the higher capacity observed in PIL-based electrolytes compared to the AIL-based one.

Finally, also the cycling stability of the LVP electrodes in the electrolytes $0.5 \mathrm{M}$ LiTFSI in PYR $\mathrm{HH}_{\mathrm{HH}}$ TFI and 0.5 M LiTFSI in $\mathrm{PYR}_{\mathrm{H} 4}$ TFSI was investigated. The tests were carried out at $30{ }^{\circ} \mathrm{C}$ using a C-rate equal to $1 \mathrm{C}$. As shown in Fig. 7, the LVP electrodes display high capacity, between 120 and $130 \mathrm{~mA} \mathrm{~h} \mathrm{~g}^{-1}$, in both PIL-based electrolytes. These values are in agreement with those observed during the C-rate test and are comparable with those shown by the LVP electrode in conventional electrolytes. ${ }^{16}$ As indicated in the figure, such high capacity can be fully maintained for 100 cycles and it is important to notice that the efficiency of the charge-discharge process was close to $100 \%$ during all cycles. These results clearly indicate that systems containing dry PIL-based electrolytes can also display high cycling stability.

\section{Conclusions}

Protic ionic liquids display all typical and favourable properties of ILs, but they have the advantage of being easier to synthesize and cheaper compared to aprotic ionic liquids. In this work, we considered two pyrrolidinium-based PILs, PYR ${ }_{\mathrm{HH}}$ TFSI and $\mathrm{PYR}_{\mathrm{H} 4}$ TFSI, in view of their use as electrolytes for LIBs. We showed that the (dry) electrolytes $0.5 \mathrm{M}$ LiTFSI in PYR $\mathrm{HH}_{\mathrm{H}}$ TFSI and 0.5 M LiTFSI in PYR $_{\mathrm{H} 4}$ TFSI display conductivity, viscosity and lithium-ion self-diffusion coefficient comparable to those of a pyrrolidinium-based AIL. However, they have the important advantage of displaying an improved performance of LVP-based electrodes during tests at a high C-rate. The lithium ions in PILbased electrolytes do not move faster than in AIL-based electrolytes due to their self-diffusion coefficients. However, fewer TFSI $^{-}$anions form the solvation sphere of $\mathrm{Li}^{+}$in the investigated PILs. We showed that the improved performance of LVP electrodes in the PIL-based electrolytes is related to a reduced charge-transfer resistance at the LVP-electrolyte interface. Also an increased $\mathrm{Li}^{+}$mobility in the presence of an electric field in the PILs compared to the one in the AIL (which is not reflected by the self-diffusion coefficients) could play a significant role and investigations about the latter point are currently in progress. Taking into account that the limited performance at a high rate of IL-based LIBs is presently considered as one of the main limitations of these devices, the use of PIL-based electrolytes can be regarded as a new and promising strategy to overcome this drawback. Additionally, since PILs are typically cheaper than AILs, the introduction of this innovative electrolyte could also be of importance for the development of safe and cheaper IL-based lithium-ion batteries.

\section{Acknowledgements}

The authors wish to thank the Westfälische Wilhelms-Universität Münster, the Ministerium für Innovation, and Wissenschaft und Forschung des Landes Nordrhein-Westfalen (MIWFT) for the financial support.

\section{Notes and references}

1 B. Scrosati and J. Garche, J. Power Sources, 2010, 195, 24192430.

2 M. Armand, F. Endres, D. R. MacFarlane, H. Ohno and B. Scrosati, Nat. Mater., 2009, 8, 621-629.

3 A. Balducci, S. S. Jeong, G. T. Kim, S. Passerini, M. Winter, M. Schmuck, G. B. Appetecchi, R. Marcilla, D. Mecerreyes, V. Barsukov, V. Khomenko, I. Cantero, M. I. De, M. Holzapfel and N. Tran, J. Power Sources, 2011, 196, 9719-9730.

4 J. F. Wishart, Energy Environ. Sci., 2009, 2, 956-961.

5 A. Guerfi, M. Dontigny, P. Charest, M. Petitclerc, M. Lagace, A. Vijh and K. Zaghib, J. Power Sources, 2010, 195, 845-852.

6 R.-S. Kühnel, N. Böckenfeld, S. Passerini, M. Winter and A. Balducci, Electrochim. Acta, 2011, 56, 4092-4099.

7 M. Anouti, M. Caillon-Caravanier, Y. Dridi, H. Galiano and D. Lemordant, J. Phys. Chem. B, 2008, 112, 13335-13343.

8 H. Matsuoka, H. Nakamoto, M. A. B. H. Susan and M. Watanabe, Electrochim. Acta, 2005, 50, 4015-4021.

9 M. Anouti, E. Couadou, L. Timperman and H. Galiano, Electrochim. Acta, 2012, 64, 110-117.

10 A. Brandt, J. Pires, M. Anouti and A. Balducci, Electrochim. Acta, 2013, 108, 226-231.

11 T. Yasuda and M. Watanabe, MRS Bull., 2013, 38, 560-566. 12 N. Böckenfeld, M. Willeke, J. Pires, M. Anouti and A. Balducci, J. Electrochem. Soc., 2013, 160, A559-A563.

13 S. Menne, J. Pires, M. Anouti and A. Balducci, Electrochem. Commun., 2013, 31, 39-41.

14 L. Timperman, P. Skowron, A. Boisset, H. Galiano, D. Lemordant, E. Frackowiak, F. Beguin and M. Anouti, Phys. Chem. Chem. Phys., 2012, 14, 8199-8207.

15 P. Stilbs, Prog. Nucl. Magn. Reson. Spectrosc., 1987, 19, 1-45. 16 N. Böckenfeld and A. Balducci, J. Power Sources, 2013, 235, 265-273.

17 L. Timperman, H. Galiano, D. Lemordant and M. Anouti, Electrochem. Commun., 2011, 13, 1112-1115. 
18 F. Castiglione, E. Ragg, A. Mele, G. B. Appetecchi, M. Montanino and S. Passerini, J. Phys. Chem. Lett., 2011, 2, 153-157.

19 S. Duluard, J. Grondin, J.-L. Bruneel, I. Pianet, A. Grélard, G. Campet, M.-H. Delville and J.-C. Lassègues, J. Raman Spectrosc., 2008, 39, 627-632.

20 D. Brouillette, D. E. Irish, N. J. Taylor, G. Perron, M. Odziemkowski and J. E. Desnoyers, Phys. Chem. Chem. Phys., 2002, 4, 6063-6071.

21 M. Herstedt, M. Smirnov, P. Johansson, M. Chami, J. Grondin, L. Servant and J. C. Lassègues, J. Raman Spectrosc., 2005, 36, 762-770.

22 A. Martinelli, A. Matic, P. Johansson, P. Jacobsson, L. Börjesson, A. Fernicola, S. Panero, B. Scrosati and H. Ohno, J. Raman Spectrosc., 2011, 42, 522-528.
23 M. Castriota, T. Caruso, R. G. Agostino, E. Cazzanelli, W. A. Henderson and S. Passerini, J. Phys. Chem. A, 2005, 109, 92-96.

24 J.-C. Lassegues, J. Grondin and D. Talaga, Phys. Chem. Chem. Phys., 2006, 8, 5629-5632.

25 S. Menne, T. Vogl and A. Balducci, Phys. Chem. Chem. Phys., 2014, 16, 5485-5489.

26 X. H. Rui, N. Ding, J. Liu, C. Li and C. H. Chen, Electrochim. Acta, 2010, 55, 2384-2390.

27 L. S. Plashnitsa, E. Kobayashi, Y. Noguchi, S. Okada and J.-i. Yamaki, J. Electrochem. Soc., 2010, 157, A536A543.

28 J.-M. Atebamba, J. Moskon, S. Pejovnik and M. Gaberscek, J. Electrochem. Soc., 2010, 157, A1218A1228. 